Société d'histoire de la révolution de 1848 et des

révolutions du XIXe siècle

46 | 2013

L'espace du politique en Allemagne au $\mathrm{XIX}^{\mathrm{e}}$ siècle

\title{
Dimensions transnationales de la culture politique rhénane, 1815-1848
}

James M. Brophy

\section{(Q) OpenEdition Journals}

Édition électronique

URL : http://journals.openedition.org/rh19/4445

DOI : $10.4000 /$ rh 19.4445

ISSN : $1777-5329$

Éditeur

La Société de 1848

Édition imprimée

Date de publication : 1 juin 2013

Pagination : 73-93

ISSN : 1265-1354

Référence électronique

James M. Brophy, «Dimensions transnationales de la culture politique rhénane, 1815-1848 », Revue d'histoire du XIXe siècle [En ligne], 46 | 2013, mis en ligne le 01 juin 2016, consulté le 01 mai 2019. URL : http://journals.openedition.org/rh19/4445; DOI : 10.4000/rh19.4445 


\section{JAMES M. BROPHY}

\section{Dimensions transnationales de la culture politique rhénane, 1815-1848}

En avril 1843, Karl Heinrich Hermes, journaliste au Kölnische Zeitung, présente sa vision des relations entre la Rhénanie et la France à Friedrich Wilhelm Saint-Paul, censeur prussien :

"Les institutions politiques françaises et belges ont involontairement une fonction de modèle pour le Rhénan, qui attend le plus grand salut de leur reproduction chez lui. Il ne souhaite pas l'union avec la France, car tous les souvenirs de cette relation malheureuse ne sont pas encore enterrés. Mais il ne voit pas pourquoi il ne devrait pas jouir de la même 'liberté' que le Français ou le Belge. À chaque fois qu'on attise en lui l'espoir d'un progrès politique, il pense être en passe d'atteindre son aspiration à l'égalité de tous les droits politiques avec les peuples voisins. C'est pourquoi le gouvernement ne doit en aucun cas espérer satisfaire profondément la Rhénanie s'il n'a pas l'intention de la doter d'une constitution ${ }^{1}$.

Cette remarque reflète le degré de politisation de la Rhénanie, mais révèle aussi un point de vue plus profondément enraciné dans les décennies suivant la domination française. Y apparaitt une dimension singulière de la francophilie rhénane : les Rhénans n'aspirent pas vraiment à être Français, mais désirent plutôt la liberté politique dont jouissent les Français, les Belges et les citoyens d'autres pays d'Europe occidentale. La recherche d'une «égalité de tous les droits politiques avec les pays voisins» renvoie à une identité politique fondée sur l'expérience européenne et non sur un modèle uniquement allemand. Pour Hermes, la Rhénanie est irrévocablement mêlée à la vie politique et culturelle de l'Europe occidentale.

Si l'on fait l'examen des facteurs de politisation et de démocratisation de l'Allemagne au XIX ${ }^{\mathrm{e}}$ siècle, le rôle de la Rhénanie apparaît évident et dominant, à travers le lien qu'elle crée entre les pratiques politiques et sociales occidentales et la culture politique allemande. La question du transfert culturel entre la Rhénanie et l'Europe de l'ouest n'est en rien un objet nouveau de

\footnotetext{
* Traduit de l'Anglais (États-Unis) par Xavier Blandin.

1. Karl Heinrich Hermes à Saint-Paul, 22 avril 1843, in Joseph Hansen [dir.], Rheinische Briefe und Akten zur Geschichte der politischen Bewegung 1830-1850, Reprint, Düsseldorf, Droste, 1997, I, p. 517.
} 
la recherche historique : il y a plus de 70 ans, Lucien Febvre plaçait le pays rhénan au carrefour de l'Europe, aux frontières politiques et économiques souples et poreuses ${ }^{2}$. Au cours des vingt dernières années, des chercheurs tels que Bernd Kortländer, Michel Espagne et Matthias Middell ont développé les concepts de «transfert» culturel et d' «entremêlement» pour penser le processus de re-localisation des idées et des impulsions ${ }^{3}$. Dans le champ de l'histoire politique, Elisabeth Fehrenbach, Karl-Georg Faber, Horst Lademacher et Klaus Pabst ont depuis longtemps reconnu l'importance de la porosité frontalière et des échanges entre la France et la Belgique ${ }^{4}$. Enfin, des historiens de l'économie comme Sidney Pollard, Herbert Kisch, Hermann Kellenbenz, entre autres, ont exploré les articulations transnationales de l'activité économique, du développement et de la coopération dans l'Europe du Nord-ouest ${ }^{5}$. Cet article entreprend donc une exploration de la politisation transnationale dans le sillage d'une riche historiographie.

Il s'appuie sur la notion d' "espace intermédiaire» de Philipp Ther et sa description de la nature mutuellement constitutive de la différence nationale, qui permettront de placer la Rhénanie au sein des arènes de communication, des réseaux sociaux, et des arrière-pays économiques plus vastes de l'Europe

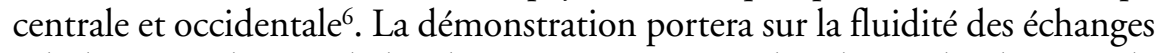
- l'identité politique de la Rhénanie restant irrésolue durant la plus grande partie du XIX ${ }^{e}$ siècle. Par ailleurs, cet article ne suit pas les anciens modèles relatifs à la modernité politique, qui voulaient voir le progrès marcher d'ouest en est, et hiérarchisaient les espaces selon leur maturité et leur développement politiques. Le simple fait que les formes polonaise, tchèque et hongroise du progressisme aient enrichi le discours politique allemand démontre l'invalidité de l'hypothèse de flux à direction unique. Ces précautions émises, la présente étude n'en cherchera pas moins à trouver une "grammaire commune de l'expérience sociale» entre la Rhénanie et ses voisins occidentaux,

2. Lucien Febvre, Le Rhin, Paris, Armand Colin, 1935.

3. Michel Espagne, Les transferts culturels franco-allemands, Paris, Presses universitaires de France, 1999; Michel Espagne et Matthias Middell [dir.], Von der Elbe bis an die Seine. Kulturtransfer zwischen Sachsen und Frankreich im 18. und 19. Jahrhundert, Leipzig, Leipziger Universitätsverlag, 1999; Bernd Kortländer [dir.], Französische Literatur in deutscher Sprache, Düsseldorf, Droste Verlag, 1986.

4. Elisabeth Fehrenbach, Traditionelle Gesellschaft und revolutionäres Recht. Die Einführung des Code Napoléon in den Rheinbundstaaten, Göttingen, Vandenhoeck \& Ruprecht, 1974; Karl-Georg Faber, Die Rheinlande zwischen Restauration und Revolution. Probleme der rheinischen Geschichte von 1814 bis 1848 im Spiegel der zeitgenössischen Publizistik, Wiesbaden, Franz Steiner, 1966; Horst Lademacher, Der europäische Nordwesten. Historische Prägungen und Beziehungen: Ausgewählte Aufsätze, Münster, Waxmann, 2001; Klaus Pabst, Eupen-Malmedy in der belgischen Regierungs- und Parteienpolitik, 1914-40, Aachen, Aachener Geschichtsverein, 1976.

5. Sidney Pollard, Peaceful Conquest: The Industrialization of Europe, 1760-1970, Oxford, Oxford University Press, 1981; Herbert Kisch, From Domestic Manufacture to Industrial Revolution: The Case of the Rhineland Textile Districts, New York et Oxford, Oxford University Press, 1989; Hermann Kellenbenz, The Rise of the European Economy: an Economic History of Continental Europe from the Fifteenth to the Eighteenth Century, New York, Holmes \& Meier, 1976.

6. Philipp Ther, 'Beyond the Nation: The Relational Basis of a Comparative History of Germany and Europe', Central European History 36 (2003), p. 45-73. 
afin d'explorer l'impact des influences transnationales sur la culture politique allemande ${ }^{7}$.

L'article adopte ainsi une approche synoptique des différentes dimensions de la politisation transnationale. Un regard porté sur le droit rhénan, indice le plus évident de la transnationalisation, sera suivi d'un examen de de la porosité des frontières de la langue, de la politique populaire, de l'imprimerie, de la presse, et de la religion. En soulignant l'ampleur de la dimension transnationale dans la culture politique rhénane au XIX ${ }^{\mathrm{e}}$ siècle, cette étude met en évidence le rôle central des échanges interculturels pour l'interprétation des idéaux politiques et des compétences civiques en Allemagne.

On se gardera ici d'adopter des définitions trop étroites des cultures politiques bourgeoise et plébéienne, pour mieux se demander comment la majeure partie de la société allemande (les artisans, les fermiers, les ouvriers, les commerçants) a pu acquérir des idéaux citoyens et participer à la construction d'une nation politique. Cette étude s'appuie pour cela sur une historiographie née dans les années 1960 et arrivée à maturité dans les années 1980, qui a permis de comprendre, à travers le pouvoir de la culture et de la communication, comment des groupes socioéconomiques variés se sont unis autour d'idées partisanes communes et de revendications de droits politiques ${ }^{8}$. Les méthodes de l'histoire sociale française et anglo-américaine présentent des outils mieux adaptés à l'exploration de la fluidité et de la flexibilité des pratiques culturelles que celles de l'historiographie allemande, dont les modèles et questionnements ne s'attachent pas aux champs culturels ni aux médias qui ont participé à l'éducation politique des Allemands. Cette impasse sur la communication est particulièrement malheureuse dans les cas du projet de recherche de Bielefeld sur Bürgertum ${ }^{9}$ et des multiples thèses

7. Rogers Brubaker, "Beyond Identity", Ethnicity without Groups, Cambridge (Mass.), Harvard University Press, 2004, p. 50.

8. Edward P. Thompson, The Making of the English Working Class, London, Victor Gollancz, 1963; Raymond Williams, Culture and Society, 1780-1950, New York, Columbia University Press, 1983; Eric Hobsbawm et Terence Ranger, The Invention of Tradition, Cambridge, Cambridge University Press, 1983; Edward Berenson, Populist Religion and Left-wing Politics in France, 1830-1852, Princeton (NJ.), Princeton University Press, 1984; Lynn Hunt, Politics, Culture, and Class in the French Revolution, Berkeley and Los Angeles (Cal.), University of California Press, 1984; Mona Ozouf, La fête révolutionnaire, 1789-99, Paris, Gallimard, 1984; Keith Baker (ed.), The French Revolution and the Making of Modern Political Culture, Oxford, Pergamon Press, 1987-1994, 4 vols.; Arlette Farge, Dire et mal dire: de l'opinion publique an XVIII siècle, Paris, Seuil, 1992; David Waldstreicher, In the Midst of Perpetual Fetes: The Making of American Nationalism, 1776-1820, Chapel Hill (NC.), University of North Carolina Press, 1997; Dror Wahrman and Colin Jones (eds), The Age of Cultural Revolutions: Britain and France, 1750-1820, Berkeley and Los Angeles (Cal.), University of California Press, 2002; Leora Auslander, Cultural Revolutions: Everyday Life and Politics in England, North America, and France, Berkeley and Los Angeles (Cal.), University of California Press, 2009.

9. Ce projet s'étend sur plus de vingt volumes et rassemble une grande variété de thèses et d'approches. Pour un résumé des résultats, $c f$. Peter Lundgreen [dir.], Sozial- und Kulturgeschichte des Bürgertums. Eine Bilanz des Bielefelder Sonderforschungsbereichs (1986-1997), Göttingen, Vandenhoeck \& Ruprecht, 2000. Pour les monographies, cf. Rudolf Boch, Grenzenloses Wachstum? Das rheinische Wirtschaftsbürgertum und seine Industrialisierungsdebatte 1814-1857, Göttingen, Vandenhoeck \& Ruprecht, 1991; Monika Wienfort, Monarchie in der bürgerlichen Gesellschaft. Deutschland und England von 1640 bis 1848, Göttingen, Vandenhoeck \& Ruprecht, 1993; Thomas Mergel, Zwischen Klasse und Konfession. 
du projet de Lothar Gall sur la Stadtbürgertum au XIX siècle, l'un comme l'autre produisant des travaux d'une qualité remarquable ${ }^{10}$. Leurs thèses respectives sur la bourgeoisie comme formation culturelle et sur la continuité historique de la bourgeoisie urbaine ne permettent pas de saisir comment certains aspects de la culture politique bourgeoise ont séduit des groupes plébéiens, lesquels se sont approprié certains éléments de la citoyenneté du droit et les ont mêlés à des répertoires revendicatifs traditionnels ${ }^{11}$. La société civile est pourtant un concept plus large que la seule bourgeoisie comme classe sociale, et ce sont ces processus de fusion, d'hybridation et de popularisation - des processus tout à la fois culturels et socioéconomiques - qui appellent une attention particulière. En adoptant un cadre interprétatif plus large de la culture politique moderne, les historiens peuvent mieux reconstituer l'amalgame complexe de facteurs qui a façonné les multiples publics politiques de l'ère napoléonienne aux révolutions de 1848-49.

\section{INSTITUTIONS RHÉNANES}

L'impact du Code Napoléon sur la société allemande est l'un des plus célèbres cas de transferts dans les relations franco-allemandes modernes. L'influence de la loi napoléonienne sur les champs juridique, commercial et politique de l'ouest de l'Allemagne n'échappa pas à l'attention de ses contemporains, pas plus qu'à celle des juristes et des historiens postérieurs ${ }^{12}$. La décision de l'État prussien en 1818 de conserver le Code civil de 1804 et le Code du commerce de 1808 est une concession pragmatique à ses provinces occi-

\footnotetext{
Katholisches Bürgertum im Rheinland 1794-1914, Göttingen, Vandenhoeck \& Ruprecht, 1994; Manfred Hettling, Politische Bürgerlichkeit. Der Bürger zwischen Individualität und Vergesellschaftung in Deutschland und der Schweiz von 1860 bis 1918, Göttingen, Vandenhoeck \& Ruprecht, 1999; Rebekka Habermas, Frauen und Männer des Bürgertums. Eine Familiengeschichte, Göttingen, Vandenhoeck \& Ruprecht, 2000; Frank-Michael Kuhlemann, Bürgerlichkeit und Religion. Zur Sozial- und Mentalitätsgeschichte der evangelischen Pfarrer in Baden 1860-1914, Göttingen, Vandenhoeck \& Ruprecht, 2001. 10. Pour des travaux représentatifs du projet Stadtbürgertum de Gall, $c f$. Hans-Werner Hahn, Altständisches Bürgertum zwischen Beharrung und Wandel. Wetzlar 1689-1870, München, Oldenbourg, 1991; Ralf Roth, Stadt und Bürgertum in Frankfurt am Main. Ein besonderer Weg von der ständischen zur modernen Bürgergesellschaft 1760-1914, München, Oldenbourg, 1996; Karin Schambach, Stadtbürgertum und industrieller Umbruch. Dortmund 1780-1870, München, Oldenbourg, 1996; Gisela Mettele, Bürgertum in Köln 1775-1870. Gemeinsinn und freie Association, München, Oldenbourg, 1998; Frank Möller, Bürgerliche Herrschaft in Augsburg 1790-1880, München, Oldenbourg, 1998; Susanne Kill, Bürgertum in Münster 1770-1870. Bürgerliche Selbstbestimmung im Spannungsfeld von Kirche und Staat, München, Oldenbourg, 2001.

11. Pour un commentaire remarquable de ces projets de recherche, $c f$. Jonathan Sperber, "Bürger, Bürgertum, Bürgerlichkeit, bürgerliche Gesellschaft: Studies of the German (Upper) Middle Class and its Sociocultural World", Journal of Modern History 69 (1997), p. 271-297.

12. Fehrenbach, Traditionelle Gesellschaft..., op. cit.; Karl-Georg Faber, Recht und Verfassung. Die politische Funktion des rheinischen Rechts im 19. Jahrhundert, Köln, Wienand, 1970; Joachim Deeters et al. [dir.], Die Französischen Jahre, Köln, Historisches Archiv, 1994; Michael Rowe, From Reich to State: The Rhineland in the Revolutionary Age, 1780-1830, Cambridge, Cambridge University Press, 2003; 200 Jahre Code civil im Rheinland, Koblenz, Verlag des Landesarchivs Rheinland-Pfalz, 2005; Kerstin Theis und Jürgen Wilhelm [dir.], Frankreich am Rhein. Die Spuren der, Franzosenzeit' im Westen Deutschlands, Köln, Greven, 2009.
} 
dentales, pour faciliter une intégration encore malaisée. Les Rhénans préféraient ces codes à l'Allgemeines Landrecht de Prusse (1794), qui maintenait les privilèges accordés à la noblesse, n'offraient pas une protection suffisante aux échanges commerciaux, et reconnaissaient encore les corporations d'artisans. Ils préféraient au contraire la loi française sur la propriété, la constitution de sociétés, la séparation de l'État et de l'Église, et la liberté de mouvement des biens et des individus. Par-dessus tout, ils étaient attachés au système du jury pour les tribunaux civils et pénaux, ainsi qu'aux dispositions du Code pour les procédures orales et la responsabilité publique. De même, marchands et capitalistes rhénans soutenaient les Chambres de commerce prévues par la loi et l'efficacité des tribunaux de commerce pour le règlement rapide et équitable des différends. Dès lors, il n'est pas étonnant que, dès 1814, le Code Civil ait été renommé Rheinisches Recht, intégré à la Rheinische Institutionen, et défendu avec ténacité au cours d'un combat politique de plusieurs décennies, première manifestation de l'Identitätsstiftung régionale ${ }^{13}$. En dehors de la Prusse, le Palatinat bavarois, la Hesse rhénane et le Grand-duché de Bade maintinrent aussi des éléments du droit français, et permirent à leurs citoyens de défendre leurs droits devant des jurys d'assises. C'est notamment pour cette raison que de nombreux écrivains prirent contact avec des imprimeurs de Bade ou du Palatinat durant le Vormärz - les jurys étant plus indulgents en cas de délit politique.

Ces aspects ont, pendant longtemps, dominé les interprétations de la culture politique rhénane, mais le Code civil est à l'origine d'un éventail beaucoup plus large d'attitudes et de comportements. Comme le remarque très justement Jonathan Sperber, la relation civique née des rapports de propriété définis par le Code civil s'étend à une vaste gamme de groupes sociaux, dont les relations matérielles ont une influence sur de nombreux autres rapports sociaux (mariage, crédit, imposition et gouvernement local) qui définissent ensemble un ordre progressiste au $\mathrm{XIX}^{\mathrm{e}}$ siècle $^{14}$. Les normes de comportement, les statuts socioéconomiques et les idéaux d'indépendance ont tous leurs racines dans la capacité à posséder et à échanger des biens. Les «régimes de propriété» de l'Allemagne de l'Ouest et du Sud-ouest, comparables à ceux de la Hollande, de la Belgique, de la France et d'autres pays du bassin atlantique, sont une dimension fondamentale (quoique peu étudiée) de la politisation du Vormärzallemand. L'abolition des restrictions d'Ancien régime sur la propriété permet l'apparition d'un nouveau type de propriétaires terriens extérieurs à l'élite, dont le statut économique, social et légal affecte la représentation de la citoyenneté ${ }^{15}$. Lorsque les Décrets de Carlsbad

13. Walter Rummel, „Das Nachwirken der französischen Herrschaft im preussischen Rheinland des 19. Jahrhunderts", in Theis et Wilhelm [dir.], Frankreich am Rhein..., op. cit., p. 138.

14. Jonathan Sperber, Property and Civil Society in South-Western Germany, 1820-1914, Oxford, Oxford University Press, 2005, passim, p. 236-239.

15. James M. Brophy, "The End of the Economic Old Order: The Great Transition, 1750-1860", in 
et le Wiener Schlussakte accélèrent le développement d'une politique constitutionnelle, les Allemands de l'Ouest et du Sud-ouest sont d'autant plus attachés au Code Civil, fondement d'un constitutionnalisme à venir. En 1842, le Kölnische Zeitung définit ainsi son credo politique :

"Cette égalité dans les devoirs et honneurs, cette unité véritable de la bourgeoisie et cette victoire, enfin générale, de la liberté civile sur le servage et la servitude héréditaire, qui ne pouvait auparavant être déclarée complète [...], cette égalité est la propriété précieuse de la Rhénanie depuis 45 ans; elle en est consciente et fière; c'est une profession de foi politique ${ }^{16}$.

L'identification de la Kölnische Zeitung avec une société civile bourgeoise encadrée par le Code Napoléon reflète non seulement le soutien du journal au libéralisme politique, mais aussi son admiration pour les radicaux et les démocrates. En 1830, la première publication de Jakob Venedey rend hommage à l'institution du jury dans la province rhénane; en 1843, Arnold Ruge souligne avec insistance les affinités des sociétés française et rhénane, et tout au long des années 1840, Karl Marx fait l'éloge à double tranchant des institutions juridiques napoléoniennes ${ }^{17}$.

\section{DES FRONTIÈrES SOUPLES}

Au-delà du cadre juridique, la question des frontières "souples» et "poreuses», et des rencontres qu'elles permettent entre les communautés voisines, doit être évoquée. Les populations frontalières sont-elles politisées par leur proximité quotidienne avec des groupes vivant autrement leur citoyenneté, et quelles sont les pratiques politiques qui émergent de ces rencontres? Au niveau de la "culture populaire ${ }^{18}$ et des pratiques extérieures aux élites,

Helmut Walser Smith (ed.), The Oxford Handbook of Modern German History, Oxford, Oxford University Press, 2011, p. 183.

16. Cité par Rummel, „Das Nachwirken“..., loc. cit., p. 131.

17. Jakob Venedey, Das Geschwornengericht in den preussischen Rheinprovinzen, Köln, Gerhard Pappers, 1830 ; anon. [Arnold Ruge], „Vorwort zur Verständigung der Deutschen und Franzosen von einem Deutschen Publizisten in der Fremde“, in Louis Blanc's Geschichte der zehn Jahre von 1830 bis 1840, trans. Gottlob Fink, Zïrich und Winterthur: Verlag des literarischen Comptoirs, 1843, xiv; Karl Marx, "Über Geschwornengerichte. Fußnote der Redaktion der Rheinischen Zeitung", Rheinische Zeitung, $\mathrm{n}^{\circ}$ 6, 6 janvier 1843, in MEGA 1er janvier, Berlin 1975, p. 391.

18. La "culture populaire» est une expression difficile à manier, et qui peut être la source d'une certaine confusion. Il ne s'agit pas ici des traditions indigènes authentiques d'avant la modernité, dont on peut raisonnablement penser qu'elles existent aux $\mathrm{XVI}^{e}$ et XVII ${ }^{e}$ siècles. La "culture populaire" désigne plutôt, dans ces pages, plus largement, les pratiques socioculturelles vécues par les Rhénans "ordinaires" de l'ère moderne. La cohabitation et la fusion des cultures de l'élite et du peuple (du textuel et de l'oral) sont monnaie courante au XVIII siècle, et il est certain que le goût de la lecture comme les économies de marché de la fin du siècle n'ont fait que renforcer cette hybridation culturelle des idées et des activités. Cf. Peter Burke, Popular Culture in Early Modern Europe, New York, Harper, 1978; Roger Chartier, The Cultural Uses of Print in Early Modern France, trans. Lydia G. Cochrane, Princeton (NJ.), Princeton University Press, 1987; Wolfgang Kaschuba, Volkskultur zwischen feudaler und bürgerlicher Gesellschaft. 
la recherche est, nous semble-t-il, trop rudimentaire pour que soient proposées des conclusions générales. Aussi fructueux et concis que soit le projet "Zwischen Maas und Rhein" des années 1990, il ne s'est guère intéressé aux cultures politiques et aux transferts culturels ${ }^{19}$. De même, l'étude rigoureusement étayée de Sebastian Scharte sur les zones frontalières d'EupenMalmedy nous apprend beaucoup sur l'acquisition des identités nationales, mais beaucoup moins sur les mécanismes de politisation et d'acquisition de la citoyenneté2 ${ }^{20}$. Les recherches les plus notables sont peut-être celles de Jörg Engelbrecht, ses études sur les rapports culturels et linguistiques entre la basse Rhénanie et les plaines étant particulièrement utiles ${ }^{21}$. Une étude spécifique de la culture politique hybride des régions frontalières de Rhénanie manque encore à la recherche historique; on aimerait y voir une description de la manière dont les structures économiques et les affinités culturelles ont permis aux populations de frontières de partager et d'échanger des valeurs politiques.

L'industrie textile peut offrir un exemple de transnationalité. Les fileurs, tisserands, fermiers, tondeurs et autres travailleurs qualifiés des industries de la laine, du lin, de la soie et du coton voient peu de différences, en termes de paie et de conditions de travail, entre les activités proto-industrielles du Brabant, des Flandres et des régions du Bas-Rhin. Parce que les villageois de langues flamande, wallonne, hollandaise, allemande et bas-allemande pratiquent les mêmes activités de filature, tissage et finition des tissus, ils reçoivent tous la même paie et subissent les mêmes conditions de travail de la part des mêmes marchands capitalistes. De plus, puisque la mécanisation atteint tôt cette région, le rassemblement de ces travailleurs dans les fabriques de Verviers, Eupen et Aix-la-Chapelle informe la culture politique populaire de la région. Il n'est pas surprenant que lors des émeutes suivies de destructions de machines à Eupen en 1821, des centaines de travailleurs de Liège

Zur Geschichte eines Begriffes und seiner gesellschaftlichen Wirklichkeit, Frankfurt a.M., Campus, 1988; idem, Lebenswelt und Kultur der unterbürgerlichen Schichten im 19. und 20. Jahrhundert, München, Oldenbourg, 1990; Robert Muchembled, Culture populaire et culture des élites dans la France moderne (XV-XVIII" siècles), Paris, Flammarion, 1978; Rolf Engelsing, "Die Perioden der Lesergeschichten in der Neuzeit. Das statistische Ausmaß und die soziokulturelle Bedeutung der Lektüre ", Archiv für Geschichte des Buchwesens 10 (1970), p. 944-1022.

19. Franz Irsigler [dir.], Zwischen Maas und Rhein. Beziehungen, Begegnungen und Konflikte in einem europäischen Kernraum von der Spätantike bis zum 19. Jabrhundert. Versuch einer Bilanz, Trier, Kliomedia, 2006; Christine Mayr, Zwischen Dorf und Staat. Amtspraxis und Amtsstil französischer, luxemburgischer und deutscher Landgemeindebürgermeister im 19. Jahrhundert, Frankfurt a.M., Peter Lang, 2006; Ruth Dörner, Staat und Nation im Dorf. Erfahrungen im 19. Jahrhundert: Frankreich, Luxemburg, Deutschland, München, Martin Meidenbauer, 2006. Les remarques de Dörner sont particulièrement pertinentes; $c f$. p. 48 et sq, 61-94.

20. Sebastian Scharte, Preussisch-deutsch-belgisch: Nationale Erfahrung und Identität. Leben an der deutsch-belgischen Grenze im 19. Jahrhundert, Münster, Waxmann, 2010.

21. Jörg Engelbrecht, Räume und Grenzen in historischer Perspektive: Die Entwicklung der deutsch-niederländischen Grenze im Wandel der Jahrhunderte, Viersen, Müser, 1997; idem, "Konfessionsbedingte Migrations- und Kommunikationsprozesse im nordwesteuropäischen Raum vom 16. zum 18. Jahrhundert: Köln, Antwerpen, Amsterdam», Blätter für deutsche Geschichte 137 (2000), p. 1-15; idem, "Verkehrs- und Kommunikationsbeziehungen zwischen Köln und dem Niederrhein ", in Dieter Geuenich [dir.], Köln und die Niederlande in ihren historischen Raumbeziehungen (15.-20. Jahrhundert), Pulheim, Rheinland-Verlag, 2000. 
se soient montrés solidaires. De la même manière, plus de 6000 sujets de Malmedy et Eupen ont participé à la rébellion de Verviers, le 29 août $1830^{22}$. La communication orale entre les ouvriers de la région d'Aix-la-Chapelle, Burtscheid et Verviers a contribué à la politisation des trois jours d'émeute d'Aix-la-Chapelle en août 1830, où apparurent des drapeaux tricolores et des mots d'ordre issus des révolutions belge et française. Ce sont très probablement de telles rencontres et de telles expériences - l'aspect "situationnel » que dégage la sociologie cognitive - qui modifient les perspectives politiques. La population migrante des années 1830 et 1840 est encore perçue comme un problème par les fonctionnaires de l'administration prussienne, qui font régulièrement état de la participation de sujets prussiens à des rites nationaux belges et hollandais, et déplorent d'autres formes de fraternisation. En 1840, la ville d'Aix-la-Chapelle enregistrait une population flottante de 3100 travailleurs immigrants, qui devaient être délibérément exclus des listes électorales de $1848^{23}$. En février de la même année, le gouvernement prussien ferme la frontière pour se protéger de la révolution, mais les Rhénans des zones frontalières connaissent depuis longtemps les idées et les symboles des droits politiques et de la révolution ${ }^{24}$.

Les documents officiels traitant des risques liés à la désertion militaire révèlent d'autres traces de communication et de sociabilité transfrontalières. Dans ces régions de Prusse, la question de la discipline militaire apparaît en 1816 , et reste prégnante jusque dans les années $1830^{25}$. Pour les fonctionnaires prussiens, les contacts des soldats étrangers avec les recrues du royaume constituent un problème récurrent. Les soldats belges et français fraternisent dans les tavernes allemandes; un rapport fait état de treize soldats belges à Heinsberg buvant dans une taverne et arborant des cocardes ${ }^{26}$. Inversement, le Landrat de Sarrelouis rapporte en 1832 que de nombreux

22. Anne-Marie Haase, „Das “Unruhige Dreieck” Aachen-Stolberg, Verviers und Eupen im Vormärz“, in Guido Müller et Jürgen Herres [dir.], Aachen, die westlichen Rheinlande und die Revolution von 1848/49, Aachen, Shaker Verlag, 2000, p. 58; Herbert Ruland [dir.], "Gott segne die Arbeit». Ein Lesebuch zur Geschichte Eupener Arbeiterschaft in französischer und preussischer Zeit (1792-1910), Aachen, Alano, 1988; idem, „Die Revolutionen von 1830 und 1848 im deutsch-belgischen Grenzland in der Tradition vorheriger Ereignisse“, in Geschichtswerkstatt [dir.], Die Revolution hat Konjunktur. Soziale Bewegungen, Alltag und Politik in der Revolution 1848/49, Münster, Westfälisches Dampfboot, 1999, p. $169-190$.

23. Jürgen Herres, „Wer ging am 1. Mai 1848 in Aachen zur ersten demokratischen Wahl? “in Müller et Herres [dir.], Aachen, die westlichen Rheinlande und die Revolution von 1848/49..., op. cit., p. 187, 193. 24. Sur l'hybridisation des frontières rhénanes, $c f$. James M. Brophy, "Which Political Nation? Soft Borders and Popular Nationhood in the Rhenish Borderlands, 1800-1849", in Marnix Beyen and Maarten Van Ginderachter (eds), Nationhood from Below: Europe in the Long Nineteenth Century, Basingstoke, Palgrave Macmillan, 2012, p. 162-189.

25. Landesarchiv Nordrhein-Westfalen (LNRW), Reg. Aachen, Nr. 1438, non paginé.

26. 5 septembre 1832, Geheimes Staatsarchiv preussischer Kulturbesitz Dahlem (GStAPK), Rep. 77, Tit. 505, n 6, Bd. III, p. 169; 18 et 24 avril 1831, General Landesarchiv Karlsruhe (GLA), Best. 236, $\mathrm{n}^{\circ} 8166$, p. 9-13; LNRW, Reg. Aachen, n 204, Bd. II, p. 417. Sur l'incident des citoyens français passant la frontière pour attaquer des soldats prussiens en 1816, $c f$. Landeshauptarchiv Koblenz (LHAK), Best. 442, n 17. Sur l'incident du 30 mai 1831 à Heinsberg, $c f$. LNRW, Reg. Aachen, n² 205, p. 36 et $s q$. 
jeunes hommes de sa région ont passé la frontière française pour y devenir conscrits $^{27}$. En 1833, des soldats d'Aix-la-Chapelle et de Heinsberg désertent aussi ${ }^{28}$. On rapporte que dans les marchés et les tavernes de Bitburg, des hommes incitent les jeunes gens à intégrer le Corps franc de Belgique, et distribuent des proclamations incendiaires ${ }^{29}$. Ces cas de désertion inquiètent d'autant plus les autorités prussiennes que plusieurs dizaines d'échauffourées éclatent entre civils rhénans et soldats prussiens, donnant au gouvernement de la région des airs d'administration coloniale ${ }^{30}$.

Des civils étrangers attirent aussi l'attention des autorités. Le flux continu de citoyens strasbourgeois dans le bourg frontalier de Korb attire dans la région d'autres Allemands «dont la sensibilité politique n'est pas compatible avec les préceptes d'un gouvernement encourageant l'ordre »31. À Cologne, le prince Wilhelm se plaint des «ouvriers belges désœuvrés qui nouent des liens avec la populace locale», et désapprouve "le comportement laxiste» des autorités qui laissent faire ces mélanges ${ }^{32}$. D'autres incidents mettent en relief la solidarité franco-rhénane contre l'autorité de l'administration, par exemple lorsque des habitants de Grande-Rosselle (près de Sarrebruck) attaquent des fonctionnaires prussiens pour libérer un voleur de bois français, amenant les officiers des forêts à exiger une escorte armée pour toute intervention future $^{33}$. Il ne s'agit pas ici de prendre le parti de la perspective officielle prussienne sur l'«influence subversive» des étrangers, mais de mieux comprendre les mécanismes d'échanges et de transferts transfrontaliers. Si la familiarité entre Belges, Prussiens et Français est attestée, il reste à préciser le degré de communication politique qui en résulte.

\section{LANGUES}

Dire que les populations frontalières communiquent entre elles, c'est soulever la question des langues. Les Rhénans ne sont pas polyglottes, et la différence linguistique est une contrainte énorme. Il ne s'agit pas pour autant de sous-estimer les échanges culturels. Côtoyer des langues étrangères n'est pas nouveau pour les Européens, et l'on pourrait facilement faire

27. 13 septembre 1832, GStAPK, Rep. 77, Tit. 505, n 5, Bd. I, p. 234.

28. GStAPK, Rep. 77, Tit. 505, n 6, Bd. II, p. 171, 193; Bd. III, p. 112, 204.

29. LHAK, Best. 403, n 2056, p. 13-15; GStAPK, Rep. 77, Tit. 505, n 5, Bd. I, p. 92-93; GStAPK, Rep. 77, Tit. 505, n 5, Bd. I, p. 81.

30. Ibid.; GStAPK, Rep. 77, Tit. 505, n6, Bd. III, p. 113; LNRW, Reg. Aachen Pr., n 643, p. 21 ; LHAK, Best. 403, n⿳ 2483, passim; LHAK, Best. 403, n² 2076, p. 1-2; LNRW, Reg. Aachen, ${ }^{\circ} 204$, Bd. II, p. 278. Sur l’"administration coloniale» prussienne en Rhénanie, $c f$. Jürgen Herres, „Und nenne Euch Preussen!» Die Anfänge preussischer Herrschaft am Rhein im 19. Jahrhundert“, in Helga Schnabel-Schüle et Andreas Gestrich [dir.], Fremde Herrscher - fremdes Volk, Frankfurt a. M., Peter Lang, 2006, p. 107-137.

31. 10 mai 1834, GLA, Best. 236, n 8776 , non paginé.

32. 14 décembre 1831, LNRW, Reg. Aachen, $n^{\circ} 242$, p. 5.

33. LHAK, Best. 403, n 2451, p. 33-39. 
valoir l'argument de Pieter Judson, Jeremy King, Tara Zahra et d'autres historiens de l'Europe du nord-ouest, selon lequel les frontières politiques correspondent rarement aux délimitations linguistiques ${ }^{34}$. La région prussienne francophone de Stablo-Malmedy, l'usage du hollandais dans les écoles et les églises de Gueldre et Cleves, tout comme les différentes gradations du basallemand, du bas-rhénan et du Niederländisch évoquent tous une certaine mixité linguistique et un certain niveau de bilinguisme qui rendent plausibles les échanges transnationaux. Il est certes probable que, malgré un quart de siècle de souveraineté française, la majorité des Rhénans n'ait jamais été bilingue. Ce fait ne doit néanmoins pas nous faire oublier la familiarité des Rhénans avec des expressions, des idées et des formules françaises. Comme le fait observer Klaus Pabst, la Rhénanie a laissé de côté son idiome de déférence courtoise (Zopfdeutsch) et intégré les expressions politiques de la Révolution dans son langage vernaculaire ${ }^{35}$. Vingt-cinq années de noms de rue en français, de monnaie française, d'almanachs, de calendriers, de placards, de décrets bilingues - tout ce que Michael Henkin appelle la «lecture de ville» - ont contribué à domestiquer la culture française ${ }^{36}$. À ce niveau d'expérience directe s'ajoute la combinaison de circonstances matérielles, sociales et culturelles qui ont produit le syncrétisme de la frontière rhénane, absorbant des éléments des cultures voisines.

Si l'Allemagne occidentale est souvent qualifiée de carrefour de l'Europe, ce constat s'applique singulièrement aux guerres napoléoniennes. Un quart de siècle de guerres et de déplacements de populations a rassemblé un grand nombre de langues, de nationalités, et de couches sociales. Sulpiz Boisserée note ainsi en 1814, dans son journal intime, à l'occasion d'un voyage en bateau de Francfort à Mayence :

«Dans les étages inférieurs du bateau, des artisans, tailleurs ou cordonniers fortunés de Francfort devisent de politique dans une petite pièce élégante à l'arrière; l'un d'eux, vêtu d'un manteau de Landsturm, pipe d'écume de mer à la bouche, parle de Napoléon avec un voyageur, un homme de troupe (sous-officier de grade inférieur), un Italien ou un Polonais. [...] Devant, dans la grande pièce, est assis un hussard anglais avec une jambe de bois. Résidant près de Creutznach, il a dû servir l'Anglais en Espagne et a perdu sa jambe à Pampelune en [18]13. Deux jeunes paysans d'une trentaine d'années et une paysanne de Darmstadt l'entourent avec compassion et

34. Pieter Judson, Guardians of the Nation: Activists of the Language Frontiers of Imperial Austria, Cambridge (Mass.), Harvard University Press, 2006; Jeremy King, Budweisers into Czechs and Germans: A Local History of Bohemian Politics, Princeton, Princeton University Press, 2002; Tara Zahra, Kidnapped Souls: National Indifference and the Battle for Children in the Bohemian Lands, 1900-1948, Ithaca (NY.), Cornell University Press, 2008.

35. Klaus Pabst, "Französisch in Verwaltung und Schule des linken Rheinufers 1792/94 bis 1814», in Bernd Spillner [dir.], Französische Sprache in Deutschland im Zeitalter der Französischen Revolution, Frankfurt a.M., Peter Lang, 1997, p. XX et $s q$.

36. David M. Henkin, City Reading: Written Words and Public Spaces in Antebellum New York, New York, Columbia University Press, 1998. 
l'écoutent debout ou assis; l'un des paysans avait été dans le [régiment de] RoyalAllemand, l'autre avait également été hussard. Ils demandent à l'invalide s'il a encore ses parents, il répond que non, et lui posent plusieurs questions ${ }^{37}$.

Boisserée montre habilement comment la circulation des nouvelles et des opinions politiques franchit les rangs sociaux aussi bien que les nationalités. L'intérêt des artisans, des paysans et de leurs épouses pour les affaires politiques semble aller de soi, tout comme la facilité avec laquelle Allemands et étrangers discutent les uns avec les autres. Cette source suggère l'existence d'une expérience commune aux Européens, au service d'une armée extérieure à leur nation. La fierté évidente des vétérans rhénans lorsqu'ils se remémorent leurs expériences dans l'armée de Napoléon n'a alors rien d'exceptionnel. La persistance de tels souvenirs allemands de la gloire française jusque dans les années 1840 et 1850 , perpétués à travers la vie associative, les fêtes de carnaval, et les ballades des chanteurs de foire, manifeste la profondeur des transferts culturels à l'œuvre ${ }^{38}$.

\section{Culture politique}

Dès lors qu'on tient pour plausible une communication transnationale au sein des espaces de l'Europe occidentale, il est possible d'éclairer d'un jour nouveau la politisation de la société rhénane, dans la mesure où idées et pratiques politiques font partie des éléments en circulation. Malgré son exclusion des réseaux formels de communication politique, la population rhénane façonne ses opinions et représentations politiques grâce à différents espaces et formes de culture populaire. Les chansons, les imprimés, la religion, les fêtes, les rituels villageois comme les échanges au marché deviennent l'occasion de communication politique pour les classes inférieures. Pour saisir l'évolution de la participation politique populaire, le rôle des idées et des pratiques transnationales apparaît essentiel. La réception allemande de l'indépendance grecque, de l'insurrection polonaise de 1830-1831, de la Révolution de Juillet, de la Fête de Hambach, et d'autres événements majeurs de l'histoire du progressisme politique, est principalement restreinte à la classe moyenne; il n'est en pas moins vrai que les classes populaires allemandes ont accès à ces événements et peuvent en débattre. Cette information et ces discussions trouvent leur origine dans l'action des imprimeurs et rédacteurs

37. Hans-Joachim Weitz [dir.], Sulpiz Boisserée, Tagebücher I, 1808-1823, Darmstadt, Eduard Roether, 1978, p. 249, 252 ; citation de Peter Fritzsche, "Specters of History: On Nostalgia, Exile, and Modernity”, American Historical Review, 106 (2001), p. 1599.

38. Walther Klein, Der Napoleonkult in der Pfalz, München, C. H. Beck, 1934; Barbara Besslich, „Die „Friedenskrankheit" und das „Erinnerungsfest". Karl Immermanns Napoleon Imaginationen und das Epigonenbewusstsein im 19. Jahrhundert", in Theis et Wilhelm [dir.], Frankreich am Rhein..., op. cit., p. 79-94. 
qui refondent les idées politiques sous forme condensée et accessible dans des almanachs, des calendriers, des prospectus, des caricatures, des chansons, des livrets de colportage et autres ${ }^{39}$. Si la majorité des historiens voit dans ces fascicules des sources tout à fait accessoires, cette étape de la transmission politique est en réalité d'une importance majeure. La prolifération de lois allemandes sur la censure s'attache à isoler les classes populaires de toute émancipation politique (l'absence de censure sur les livres de plus de 21 Bogen (feuilles), soit 320 pages, établit par contraste le droit concédé à la bourgeoisie d'accéder à l'information politique); aussi ce type de documents constitue-t-il l'une des formes les plus subversives d'imprimés politiques.

La politisation des fêtes et des marchés offre aux travailleurs et ouvriers un accès aux symboles, aux mots d'ordre, et à la culture oppositionnelle du monde post-révolutionnaire. L'incarnation de la citoyenneté de droit dans la langue, les pratiques festives, les tenues vestimentaires et les marchandises, notée tout autour de l'Atlantique par Leora Auslander, est applicable à de nombreux éléments de la société rhénane ${ }^{40}$. Porcelaines, pipes, tabatières, chapeaux, broches et autres vestes, chemises et sarraus, marquent aussi les identités politiques en Rhénanie, tout autant que la chevelure ou la diction. La loi rhénane ne punit pas l'exposition de symboles révolutionnaires ${ }^{41}$, pas plus qu'elle n'interdit la vente de souvenirs libéraux ou révolutionnaires. Lorsqu'ils statuent sur les activités politiques de la Fête de Hambach en mai 1832, les tribunaux de Bavière jugent la distribution de cocardes démagogue mais reconnaissent que leur vente est un échange contractuel légal ${ }^{42}$. La vente et l'exposition d'images - caricatures, allégories politiques et dessins satiriques - dans les librairies et les marchés est en particulier une source d'inquiétude pour les autorités. Les progrès de l'imprimerie et de la lithographie dans les années 1820 permettent de faire circuler à peu de frais une grande quantité d'images, et les imprimeurs peuvent ainsi faire face à une demande de plus en plus grande d'images à caractère politique. La douzaine d'images d'hommes politiques prussiens censurée dans les années 1830 et 1840 suggère que le marché de la représentation d'opposition est très actif ${ }^{43}$. Ce n'est

39. Sur la traduction des questions politiques dans les calendriers et almanachs populaires au cours du Vormärz, cf. James M. Brophy, "The Common Reader in the Rhineland: The Calendar as Political Primer in the Early Nineteenth Century", Past and Present 185 (2004), p. 119-157; plus généralement sur cette question : Holger Böning et Reinhart Siegert [dir.], Volksaufklärung: Biobibliographisches Handbuch zur Popularisierung aufklärerischen Denkens im deutschen Sprachraum von den Anfängen bis 1850, 3 volumes, Stuttgart/Bad Cannstadt, Frommann-Holzboog, 1990.

40. Leora Auslander, Cultural Revolutions: Everyday Life and Politics in England, North America, and France, Berkeley and Los Angeles, University of California Press, 2009. Sur l'uniformisation grandissante de cette incarnation dans le monde, $c f$. Christopher Alan Bayly, The Birth of the Modern World, 1780-1914, London, Arnold, 2004, p. 17.

41. Johannes Hauch est arrêté pour avoir tissé et brandi un drapeau tricolore en juin 1832, mais il est acquitté, le tribunal n'ayant trouvé aucune loi prévoyant une peine pour ces actions dans le Code pénal. Actes du tribunal, 8 août 1832, Landesarchiv Speyer, Best. J1, Nr. 39.

42. Session du 17 mars 1833, Landesarchiv Speyer, Best. J1, nº 39.

43. GStAPK Berlin, Rep. 77, Tit. 2, Gen. n 7, Bd. I \& II; Mary Lee Townsend, Forbidden Laughter: 
qu'avec les Six actes de 1832 que les gouvernements trouveront une réponse légale aux images séditieuses, mais les cocardes comme les drapeaux tricolores venus de France, de Belgique, de Hollande et d'Allemagne continueront pourtant de circuler en Rhénanie durant le Vormärz.

L'abondance de sources de ce genre est trop grande pour que cet article en fasse une étude plus détaillée ${ }^{44}$, mais les arbres de la liberté (Freibeitsbäume) offrent un exemple de pratique permettant aux Rhénans de fonder leur nation politique. Lorsqu'il est introduit par les Jacobins dans les années 1790, l'arbre de la liberté est encore rarement planté par les Rhénans; s'ils se mêlent au rituel, c'est principalement parce qu'ils y sont forcés ${ }^{45}$. Dans les années 1830, en revanche, de nombreuses communautés rhénanes de Prusse, de Bavière et de Bade s'approprient le rite de deux manières : d'abord pour montrer leur solidarité avec les mouvements constitutionnalistes de la monarchie de Juillet et de la Fête de Hambach, et aussi comme acte de résistance contre les incursions de l'État dans la vie des villes et villages durant le Vormärz. En 1848, lorsque les bourgs du Rhin et de la Moselle plantent des dizaines d'arbres de la liberté, le rituel fait partie intégrante du répertoire de pratiques démocratiques. Le rituel se fait sans contrainte, et ses participants sont pleinement conscients des revendications progressistes associées à leur geste. Si l'on s'attache à suivre l'histoire des arbres de la liberté sur un demi-siècle, il apparaît qu'un symbole étranger se transforme en coutume locale. Son déploiement entre 1830 et 1848 retrace l'évolution d'une grammaire politique annonçant la revendication d'une participation politique et de droits universels.

Ces questions sont aussi directement liées au rôle que jouent dans la mobilisation villageoise les politiques étatiques et l'imposition d'une discipline aux populations. La protestation des classes populaires de Rhénanie se fait en réponse à l'ingérence de l'État dans la vie des villages, amorcée sous la domination française mais de plus en plus présente avec les gouvernements de Prusse, de Bavière et de Bade. Dans tous ces cas, le besoin de liberté et d'autonomie côtoie les autres formes d'opposition politique ${ }^{46}$. C'est ce

Popular Humor and the Limits of Repression in Nineteenth-Century Prussia, Ann Arbor (MI), University of Michigan Press, 1992.

44. Pour une discussion plus poussée, $c f$. James M. Brophy, Popular Culture and the Public Sphere in the Rhineland, 1800-1850, Cambridge, Cambridge University Press, 2007.

45. Hansgeorg Molitor, Vom Untertan zum Administré. Studien zur französischen Herrschaft und zum Verhalten der Bevölkerung im Rhein-Mosel Raum von den Revolutionskriegen bis zum Ende der Napoleonischen Zeit, Wiesbaden, Steiner, 1980; Norbert Finzsch, Obrigkeit und Unterschichten. Zur Geschichte der rheinischen Unterschichten gegen Ende des 18. und zu Beginn des 19. Jahrhunderts, Stuttgart, Steiner, 1990; Tim Blanning, Reform and Revolution in Mainz, 1743-1803, Cambridge, Cambridge University Press, 1974.

46. Sur la question de la protestation populaire et du Staatlichkeit, cf. Walter Rummel, „Gegen Bürokratie, Steuerlast und Bevormundung durch den Staat. Anliegen und Aktionen der ländlichen Gebiete der Rheinprovinz während der Revolution 1848/49“, in Stephan Lennartz et Georg Mölich [dir.], Revolution im Rheinland. Veränderungen der politischen Kultur 1848/49, Bielefeld, Verlag für Regionalgeschichte, 1998, p. 109-162; Rummel, „Kanonen gegen Winzer - Kolonnen gegen Bauern. Die Revolution von 1848/49 in den ländlichen Gebieten des Saar-Mosel-Raumes“, Jahrbuch für westdeutsche 
mélange d'impulsions politiques mal définies qui rend l'interprétation de la culture politique de l'époque si difficile.

\section{LECTURE TRANSNATIONALE}

La politisation populaire en Rhénanie est directement liée à la société bourgeoise, très bien informée sur les autres cultures politiques démocratiques et constitutionnelles. Son éducation est en grande part à mettre au crédit de l'imprimerie, ce qui nous donne l'occasion de revenir sur la maxime bien connue de Benedict Anderson sur le "capitalisme d'imprimerie» et les langues vernaculaires, cause de la "fatale diversité» de l'ère moderne. Pour Anderson, les langues vernaculaires séparent et divisent, et fournissent ainsi le cadre du nationalisme d'exclusion. Les moteurs discursifs du capitalisme d'imprimerie, selon lui, permettent aux États et aux nations de créer des cultures d'adversité nationale en délimitant les visions politiques et en s'accaparant les espaces de communication.

L'argumentaire d'Anderson est sans aucun doute lumineux du point de vue conceptuel, mais il est aussi possible de se demander si les communautés parlant une seule langue sont vraiment aussi isolées qu'il le dit. La «camaraderie horizontale" du nationalisme s'étend-elle seulement aux groupes sociaux à l'intérieur des frontières - et, inversement, les communautés partageant le même imaginaire se représentent-elles automatiquement les autres nations comme ennemies? Sans douter de l'importance de la notion de capitalisme d'imprimerie, la recherche sur l'histoire de l'imprimerie suggère d'autres points de vue concernant la traduction, la transcription, la condensation, la circulation, la réimpression et la copie sans autorisation. La notion d'exclusion linguistique apparait alors trop simple.

Surtout, la "prière quotidienne" que voit Anderson dans la lecture du journal évoque la puissante idée d'un lectorat anonyme, qui viendrait renforcer le sentiment d'appartenance à une nation. Mais il est aussi possible de voir comment la presse du XIX siècle a sapé la montée des nationalismes d'exclusion. Une étude poussée de la Kölnische Zeitung et de son assignation de valeurs, ainsi que son traitement des espaces géographiques, permet de voir que les références nationales sont estompées, et que les priorités politiques de l'État-nation perdent alors une grande partie de leur impact ${ }^{47}$.

Landesgeschichte 24 (1998), p. 305-328; Walter Rummel, 'Taxes and Other Burdens: State-Building in the Prussian Rhine Province, 1815-1850', in Alexander Nützenadel et Christoph Strupp [dir.], Taxation, State, and Civil Society in Germany and the United States from the 18th to 20th Century, Baden-Baden, Nomos, 2008, p. 141-152; sur la violence entre civils et autorités durant la Vormärz, cf. James M. Brophy, "Violence between Civilians and State Authorities in the Prussian Rhineland, 1830-1846", German History 22 (2004), p. 1-35.

47. Aled Jones, 'The Dart and the Damning of the Sylvan Stream: Journalism and Political Culture in the Late-Victorian City', in Laurel Brake and Julie F. Codell (eds), Encounters in the Victorian Press: Edi- 
L'analyse que fait le quotidien de la politique en France, en Belgique ou en Hollande est bien plus riche et plus nuancée qu’à propos de Berlin, Dresde ou Vienne. Bien sûr, la censure est en grande partie responsable de la manière dont un journal progressiste allemand formule des nouvelles et des critiques sur les autres États de la Confédération, mais cette influence ne fait pas tout. La demande de reportages sur l'Europe occidentale vient moins d'un désir d'accentuer les différences que d'une volonté d'affirmer un plus grand espace d'identification. De plus, croisements et hybridations ne sont pas nécessairement des facteurs de durcissement des positions nationales, comme le voudrait Anderson.

Durant la Crise du Rhin (1840-41) par exemple, lorsque l'Allemagne se détourne du progressisme cosmopolite pour définir son nationalisme, la Kölnische Zeitung juge la politique française de façon beaucoup plus pertinente et équilibrée qu'on ne pourrait l'attendre. Le journal de Cologne révèle une culture politique ouverte à l'Ouest. Les reportages sur la France et la Belgique sont menés à grande échelle, précis et concrets; la situation géographique du quotidien lui permet de rapporter les nouvelles d'Europe occidentale avant tous ses concurrents (en 1848, le journal utilise des pigeons voyageurs pour abaisser les délais de communication à seize heures ${ }^{48}$ ). En 1840-41, la Kölnische Zeitung publie de nombreux articles de ses correspondants à Paris, avec des analyses précises de la nature mouvante et instable des factions parlementaires qui orchestrent la peur d'un conflit armé. La couverture multidimensionnelle des affaires politiques parisiennes est toujours plus rigoureuse que pour Berlin ou d'autres métropoles allemandes. Un lecteur perspicace pouvait y lire comment et pourquoi c'était la politique intérieure française qui préparait le pays au conflit, plus que les besoins militaires ou les questions diplomatiques; il pouvait y découvrir le détail des querelles partisanes, et le résumé des vues des journalistes politiques parisiens tout aussi querelleurs et désunis. Si les articles manifestent un certain mépris pour les excès de la politique partisane, ils révèlent aussi une fascination pour les subtilités de la politique représentative ${ }^{49}$. Le journal laissera même de côté la crainte de la guerre à partir de novembre 1840, pour s'intéresser à l'utilité et aux abus de la représentation politique. Le quotidien révèle, et c'est un point plus important encore, un imaginaire national et civique qui fait la part belle au réformisme à l'intérieur des frontières plutôt qu'à l'appel aux armes. Au milieu des cris et vagissements optimistes d'une démocratie naissante, la Rhénanie

tors, Authors, Readers, Basingstoke, Palgrave Macmillan, 2005, p. 177-194; Ann Ardis, 'Counter-Memories of Englishness: The Crisis's Self-Positioning in the Atlantic World', manuscrit non publié. 48. Rudolf Schmidt, Deutsche Buchhändler, deutsche Buchdrucker. Beiträge zu einer Firmengeschichte des deutschen Buchgewerbes, Reprint, Hildesheim, Georg Olms, 1979, p. 184.

49. Les références sont trop nombreuses pour être toutes citées ( $c f .1840-41$, passim), mais on verra la place centrale de la France dans «Die Ereignisse des Jahres 1840 »: Kölnische Zeitung, n 1, 5, 6, 7, 9, $10,11,12,13,14$, et 15 janvier 1841 ; des exemples de reportage en France, $c f . \mathrm{n}^{\circ} 348,12$ déc. 1840 ; $\mathrm{n}^{\circ} 357,21$ décembre $1840 ; \mathrm{n}^{\circ} 360,24$ décembre $1840 ; \mathrm{n}^{\circ} 364,28$ décembre $1840 ; \mathrm{n}^{\circ} 18,18$ janvier $1841 ; \mathrm{n}^{\circ} 19,19$ janvier 1841 et Beilage, $\mathrm{n}^{\circ} 21,21$ janvier 1841. 
prussienne fait entendre sa voix contre la censure, pour la liberté religieuse, la transformation de la Diète provinciale en corps législatif à part entière, et surtout pour que soit tenue la promesse royale d'une constitution. Dans ces pages, la réforme civique prend le pas sur la diatribe martiale ${ }^{50}$.

L'appétit rhénan pour les publications et livres français est grand; après 1840, la demande de nouvelles financières et industrielles venues de France, de Belgique et de Hollande ne fait qu'augmenter ${ }^{51}$. Sur les 189 journaux étrangers officiellement en circulation en Rhénanie prussienne en 1842, 91 sont français, remarque Jürgen Herres. Les mémoires et récits de vie suggèrent par ailleurs que les Rhénans préferent les journaux français et belges à la presse allemande, en laquelle ils avaient moins confiance ${ }^{52}$. C'est pour ces raisons que l'enthousiasme rhénan à la nouvelle du retour des cendres de Napoléon à Paris en décembre 1840 - en plein cœur de la crise - ne devrait peut-être pas nous surprendre. La Kölnische Zeitung publie un hommage chaleureux à cette occasion, et traduit même le célèbre éloge de Victor Hugo $^{53}$. La Crise du Rhin, souvent analysée comme l'un des moteurs du chauvinisme, révèle aussi un nationalisme régional où sont mis en avant les droits civiques et les libertés plus que le "racisme» et l'exclusion nationale.

Michel Espagne, parmi d'autres, a engagé avec brio la recontextualisation des idées et des auteurs dans des cadres nouveaux, et beaucoup reste à faire sur la circulation des idées politiques entre les centres d'imprimerie de France et d'Allemagne. Les circuits suivis par les imprimés ont toujours été internationaux, et les marchés du XIX ${ }^{\mathrm{e}}$ siècle n'ont fait qu'augmenter le rythme et le volume des échanges. En 1837, la firme Brockhaus \& Avenarius ouvre à Paris une maison affiliée à son entreprise de Leipzig, marquant le début d'une nouvelle ère d'échanges plus rapides et plus continus des idées et des textes, non seulement avec Paris mais aussi Édimbourg, Londres et l'Amérique du Nord $^{54}$. Les ventes allemandes d'ouvrages traduits du français atteignent une masse critique en 1770, mais s'épanouissent pleinement durant le Vormärz $z^{55}$.

50. Cf. James M. Brophy, «The Rhine Crisis of 1840 and German Nationalism: Chauvinism, Skepticism, and Regional Reception ", Journal of Modern History 85,1 (2013): 1-35.

51. Les journalistes contemporains remarquent aussi l'heureuse rencontre du commerce et du Catholicisme en Europe occidentale, qui unifie la Belgique, une partie de la Hollande, et la Rhénanie. Voir par exemple anonyme, "Belgien, Holland und die Rheinlande", in Das Katholische Europa oder Erhaltung, Fortschritt, Friede und Freiheit, Mannheim, Heinrich Hoff, 1840, p. 148-152.

52. Jürgen Herres, «Rhein-Preussen»..., op. cit., p. 193-94.

53. Cf. D. Reifferscheld's ,Napoleons Asche', Kölnische Zeitung, nº 335, 29 novembre 1840; Victor Hugo, ,Des Kaisers Wiederkehr', Kölnische Zeitung, nº 355, 19 décembre 1840; , Immermanns Aussprüche über Napoleon', Kölnische Zeitung, n . 309, 3 novembre 1840; pour une réponse au texte de Victor Hugo, cf. ,Epistel an Herrn Victor Hugo auf seine Ode am 15. December’, Kölnische Zeitung, n 365 , 29 décembre 1840. La relation complexe de la Rhénanie à Napoléon est remarquablement analysée par Barbara Besslich dans Der deutsche Napoleon-Mythos. Literatur und Erinnerung 1800 bis 1945, Darmstadt, Wissenschaftliche Buchgesellschaft, 2007.

54. Thomas Keiderling [dir.], F.A. Brockhaus, 1905-2005, Leipzig, Brockhaus, 2005, p. 188-218.

55. Jeffrey Freedman, Books without Borders in Enlightenment Europe: French Cosmopolitanism and German Literary Markets, Philadelphia (Penn.), University of Pennsylvania Press, 2012; Bernd Kortländer [dir.], Übersetzen im Vormärz, Bielefeld, Aisthesis, 2008. 
La carrière d'éditeur de Heinrich Hoff, imprimeur radical de Mannheim, donne un exemple typique des profits tirés de certains textes français. En 1835, il s'enrichit avec la traduction de L'Histoire de la Révolution de François Mignet et L'Histoire de Napoléon de Paul de Ségur, les ventes s'élevant à 40000 exemplaires pour chaque ouvrage ${ }^{56}$. Hoff publie ensuite les Mémoires de Napoléon, ceux du Maréchal Ney, Le Peuple de Michelet, et de multiples éditions de L'Histoire de la Révolution française par Adolphe Thiers. On peut ajouter à cette liste les études de Ludwig Frey sur le Code civil français, qui réaffirment la dette que doit l'Allemagne aux traditions juridiques napoléoniennes. Quelle que soit la réception précise de ces textes, Hoff procède à un transfert interculturel conscient entre la France et l'Allemagne. Ses tendances démocratiques se révèlent plus encore dans ses publications du Bürgerbuch de Hermann Püttmann et Die Opposition de Karl Heinzen, dans lesquels sont commentés Proudhon, Saint-Simon, Cabet et d'autres auteurs du socialisme et du communisme d'Europe occidentale. Hoff fait partie d'un petit groupe d'éditeurs (avec Otto Wigand, Philipp Reclam, C.W. Leske, Julius Campe, Friedrich Gottlob Franckh, etc.) pour lesquels l'attachement aux valeurs de la démocratie libérale coïncide très bien avec les profits à tirer de la traduction des textes français. Ces éditeurs établissent le lien entre le circuit de distribution de l'imprimerie et les idées et débats qui animent les cultures politiques démocratiques du monde atlantique ${ }^{57}$. Durant la première partie du XIX ${ }^{e}$ siècle, les idéaux politiques des «Lumières radicales» en Europe occidentale pénètrent aussi, et reconfigurent le paysage politique de l'espace germanophone $e^{58}$.

\section{Religion et politique}

La lecture transnationale du religieux et du politique ajoute à la compréhension des mécanismes de démocratisation et de politisation en Rhénanie. En termes topographiques simplifiés, les rythmes et les espaces culturels de la piété populaire catholique - ses pèlerinages, ses processions, ses festivals - correspondent mal à la découpe des frontières internationales. Les catholiques, lorsqu'ils traversent les frontières en pèlerinage ou au cours de

56. Il existe alors dix traductions allemandes de l'ouvrage de Mignet, $c f$. Bernd Kortländer, „Übersetzungen aus dem Französischen im Vormärz. Erkundung eines untergegangenen Kontinents" in Kortländer, Übersetzen im Vormärz..., op. cit., p. 23. Sur Gottlob Franckh, autre éditeur important de textes traduits, $c f$. Christine Haug, ,.,Der famose Ambaßadeur des künftigen freien Deutschlands revolutionären."Der Verleger Friedrich Gottlob Franckh zwischen spekulativem Unternehmertum und revolutionärem Visionen. Das Stuttgarter Buch- und Verlagsgewerbe im Vormärz", in Christian Liedtke [dir.], Literaturbetrieb und Verlagswesen im Vormärz, Bielefeld, Aisthesis, 2011, p. 103-120.

57. James M. Brophy, „Heinrich Hoff and the Print Culture of German Radicalism“, Leipziger Jahrbuch zur Buchgeschichte 19 (2010), p. 71-116.

58. Jonathan I. Israel, Radical Enlightenment: Philosophy and the Making of Modernity, Oxford, Oxford University Press, 2001. 
processions, sont conduits à aller à l'encontre de l'ordre établi par l'autorité de l'État et contre le clergé. Les problèmes disciplinaires consécutifs à des pèlerinages non autorisés de catholiques rhénans sont légion durant la première moitié du siècle. À l'occasion de l'arrestation par le gouvernement prussien, en 1837, de l'archevêque de Cologne, les catholiques rhénans se sentent profondément lésés. Dès lors, les fondements religieux des activités indépendantistes et de la désobéissance civile se manifestent de plus en plus clairement au cours de ce qui prendra le nom de "troubles de Cologne» (die Kölner Wirren). Des groupes sociaux rejetant jusque-là les principes de la représentation politique moderne font alors entendre leur voix en faveur de droits constitutionnels garantissant la liberté de religion. Les «troubles de Cologne» ont ainsi conduit les tenants du conservatisme social à revendiquer la citoyenneté de droit. Ce moment protestataire annonce le début du catholicisme politique moderne.

Pour définir leurs aspirations politiques, les catholiques rhénans s'inspirent de modèles étrangers. Les «troubles de Cologne» confirment l'attrait de la monarchie constitutionnelle belge. Les catholiques de Rhénanie admirent ainsi le compromis auquel sont parvenus Louis de Potter et Mgr van Bommel au début des années 1830, où s'équilibrent le religieux, le politique et les libertés économiques. Les institutions catholiques belges en font d'ailleurs une promotion active. Les presses de Sittard et Lüttich/Louvain publient ainsi nombre de fascicules encourageant les Rhénans à créer un État catholique. L'Église catholique belge autorise la publication de textes en allemand enjoignant les prêtres rhénans à placer l'Église universelle avant les États protestants, et les universités et lycées catholiques de Belgique bénéficient de la faveur des familles bourgeoises de Rhénanie, qui encouragent leurs enfants à venir étudier en Belgique. La crise renforce les anciennes connexions catholiques avec le Brabant, ce qui explique en partie la sympathie des factions grossdeutsch favorables à une Grande Allemagne jusque dans les années 1870.

Un autre moment transnational de la politique catholique rhénane gravite autour de la personnalité de l'Irlandais Daniel O'Connell. En 1829, le succès de la Catholic Association dans sa campagne pour obtenir l'émancipation politique des catholiques au Royaume-Uni éveille l'intérêt de la Kölnische Zeitung et d'autres publications rhénanes. O'Connell fascine ses contemporains par son mélange de nationalisme civique, de libéralisme constitutionnel et de protestation démocratique; son appel à la liberté religieuse et à la séparation de l'Église et de l'État le distingue des ultramontains du continent. Son rejet de la violence révolutionnaire et son insistance sur la réforme parlementaire lui valent l'admiration de beaucoup, même si les tenants du conservatisme social critiquent l'inféodation de son catholicisme politique aux idéologies démocratique et progressiste. De manière générale, la réception de Daniel O'Connell en Allemagne est à la fois intense et nuancée dans les années 1830 et 1840 . Son rôle dans la culture politique rhénane prend une dimension 
nouvelle quand, en novembre 1837, une semaine après l'arrestation de l'archevêque, O'Connell dénonce le roi de Prusse et le gouvernement dans un quotidien anglais, The Morning Chronicle ${ }^{59}$. Les gouvernements prussien, allemand et autrichien voient alors dans le libérateur irlandais une menace politique, et sévissent contre les collectes de fonds et les prières publiques en sa faveur. L'adulation pour l'homme politique irlandais atteint son sommet en 1843, lorsqu'il est jugé et emprisonné pendant trois mois. Des reportages quotidiens sur son sort font entrer le nom d'O'Connell dans le vocabulaire politique de la Rhénanie; en 1844, la Kölnische Zeitung publie pas moins de 22 articles sur lui ${ }^{60}$. À Coblence, l'adulation se manifeste dans certaines tavernes par l'affichage de lithographies à son effigie, auxquelles sont parfois ajoutés des lauriers ${ }^{61}$. Sa relaxe en 1844 déclenche une vague de manifestations spontanées et d'illuminations en Rhénanie, qui suggèrent la profondeur de l'impact de sa carrière politique sur ces espaces ${ }^{62}$.

Le débat intense autour de l'homme politique irlandais informe l'imaginaire politique des catholiques allemands. Alors que l'Allemagne se tient au seuil de la modernité politique, O'Connell propose une nouvelle synthèse civique. Il démontre que la confluence des pratiques associatives et de la représentation parlementaire peut conduire à des progrès, et que des catholiques peuvent pratiquer leur foi à l'intérieur d'une société respectueuse de la religion. Surtout, il ouvre la voie à une politique populaire catholique, une mobilisation de masse appuyée sur le droit au changement pacifique et à la réforme parlementaire ${ }^{63}$.

La création en 1848 du Piusverein für religiöse Freiheit (Association de Pius pour la liberté réligieuse) se fait en hommage direct à O'Connell; ses tactiques d'agitation et de propagande s'inspirent clairement de celles de l'Association catholique. Lorsque le Piusverein devient Association catholique, l'influence des principes politiques d'O'Connell est encore plus évidente. L'association rassemble prêtres et laïcs à l'aide d'imprimés incitant à la mobilisation des masses. De plus, d'éminents hommes politiques catholiques, comme Peter et August Reichensperger, Wilhelm Emmanuel von Ketteler, Franz Joseph Ritter von Buß ou Ludwig Windthorst, s'inspirent directement des pratiques d'O'Connell pour la création d'un mouvement catholique

59. Morning Chronicle, Londres, 30 novembre 1837.

60. Kölnische Zeitung, n 92, 94, 96-99, 101-103, 106, 107, 109-114, 116, 117, 118-120, avril 1844.

61. Allgemeine Zeitung, Augsburg, n 258, 14 septembre 1844.

62. Peter Alter, ,O'Connell and German Politics" ", in Maurice O'Connell [dir.], Daniel O'Connell: Political Pioneer, Dublin, 1991; Fergus O'Ferrall, Catholic Emancipation: Daniel O'Connell and the Birth of Irish Democracy, Dublin, 1985; Karl Holl, Die irische Frage in der Ara Daniel O'Connells und ihre Beurteilung in der politischen Publizistik des deutschen Vormärz, Phil. Diss. Mainz, 1958; Geraldine Grogan, The Noblest Agitator. Daniel O'Connell and the German Catholic Movement 1830-1850, Dublin, 1991.

63. James M. Brophy, «Die Rezeption Daniel O’Connells und der irischen Emanzipationsbewegung im vormärzlichen Deutschland», Marx-Engels Jahrbuch 2011, p. 74-93. 
puissant et pérenne en Allemagne ${ }^{64}$. L'influence d'O'Connell sur le Parti centriste et sur les tactiques catholiques au moment du Kulturkampf peut également être soulignée. L'éphémère Verein deutscher Katholiken (Association des catholiques allemands) de Graf Felix von Löes (1872-76) invoque directement O'Connell lorsqu'il organise des manifestations et diffuse massivement des textes de mobilisation catholique ${ }^{65}$. L'attention portée à O'Connell par les Allemands du Vormärz relève ainsi moins de la focalisation sur un individu que de la découverte d'un médiateur de mobilisations transnationales.

$*$

L'expérience rhénane suggère que la recherche historique sur l'Allemagne du XIX ${ }^{\mathrm{e}}$ siècle gagnerait à intégrer la notion de transnationalité, non pas seulement comme un outil supplémentaire, mais bien comme un élément constitutif de l'interprétation des comportements politiques modernes. Au cours du XIX e siècle, la culture rhénane s'est mêlée d'élans vers une citoyenneté de droit propres à l'ère atlantique. Si Horst Dippel et Jonathan Sperber ont raison de faire valoir que des différences fondamentales existent encore à la fin du XVIII e siècle entre les conceptions occidentale et allemande de la liberté, il est cependant nécessaire de reconnaître le rôle crucial de la Restauration et du Vormärz dans l'estompage de ces différences. La reconstitution des pratiques citoyennes dans l'histoire de l'Europe centrale s'inscrit dans de multiples dimensions, et la Rhénanie tient une place fondamentale dans cette histoire en permettant notamment de réévaluer l'ampleur sociale de la politisation. Des formes nouvelles et irrégulières de compétence et de participation politique traversent les classes sociales, et viennent redéfinir la culture politique. L'engagement partisan trouve sa place dans des textes destinés à l'élite comme aux classes populaires, mais aussi par le vecteur plus éphémère des brochures et des placards. Les structures élémentaires de la communication sociale s'en trouvent altérées : le textuel se mélange au festif, à la tradition orale, aux rites traditionnels, aux pratiques religieuses de la ville et de la campagne.

Ces vagues de politisation qui transforment la Rhénanie dans la première moitié du siècle renouvellement l'interprétation générale de l'histoire politique du XIX ${ }^{\mathrm{e}}$ siècle allemand. La politisation omniprésente des classes populaires invalide l'hypothèse selon laquelle ces dernières seraient absentes du paysage politique post-révolutionnaire. Au contraire : artisans, fermiers et ouvriers font l'apprentissage de nouvelles formes de citoyenneté à travers divers vecteurs culturels, et par leur participation élargissent l'horizon politique de l'Allemagne. Dès lors, les analyses fonctionnalistes, qui font de la

64. Grogan, Noblest Agitator..., op. cit., p. 79-98; Margaret L. Anderson, Windthorst: A Political Biography, Oxford, Oxford University Press, 1981, p. 3-4.

65. Anderson, Windthorst..., op. cit., p. 180. 
privation le seul moteur de la protestation populaire, ne convainquent guère. L'historiographie traditionnelle sous-estime l'intense politisation antérieure à la Révolution de 1848-49. L'articulation rapide de différentes factions et d'intérêts variés - conservateurs, catholiques, libéraux modérés, démocrates radicaux, socialistes - est une manifestation d'un processus de maturation déjà en cours dans les décennies précédentes. La politisation populaire du premier XIX ${ }^{e}$ siècle ne naît pas ex nihilo; l'Allemagne des débuts de l'ère moderne a déjà produit de nombreuses formes de protestation propres à des sujets ordinaires ${ }^{66}$. Mais au XIX ${ }^{\mathrm{e}}$ siècle la politique populaire excède les revendications classiques d'Ancien Régime, liées aux privilèges et droits traditionnels, et incorpore les notions de représentation constitutionnelle et de souveraineté du peuple. De plus, ces exigences ne s'éteignent pas avec le renouveau du pouvoir conservateur en 1849; comme le soulignent des études récentes, l'idéal d'une nation démocratique et constitutionnelle émis en 1848 continue d'exercer une influence considérable sur l'imaginaire politique allemand sous l'Empire. Sans perdre de vue la centralité du conservatisme prussien dans la modernité politique allemande, il importe de constater que le cadre idéologique, émotionnel et institutionnel de l'édification de la nation allemande doit aussi beaucoup à «un projet en grande partie libéral et démocratique ${ }^{67}$. C'est un point d'une importance majeure. Le retour à l'ordre post-1849 relève plus de contingences historiques que d'un Sonderweg tracé depuis longtemps ${ }^{68}$. Le potentiel de réforme politique dans l'Étatnation allemand reste ouvert, non seulement durant les Gründerjahre, mais aussi sous l'Empire. L'analyse transnationale permet de mettre au jour ces éléments participatifs et démocratiques; c'est une approche particulièrement utile pour dégager des modèles comparatistes évitant toute normativité.

James Brophy est professeur d'histoire à l'université du Delaware, États-Unis

66. Cf. Peter Blickle, Deutsche Untertanen: Ein Widerspruch, München, C.H. Beck, 1981.

67. Mark Hewitson, Nationalism in Germany, 1848-1866: Revolutionary Nation, Basingstoke, Palgrave Macmillan, 2010, p. 358.

68. David E. Barclay, «Preussens Verschwinden. Ein Streifzug durch die angloamerikanische Literatur», Zeitschrift für Ideengeschichte V/4 (2011), p. 52-64. 